\title{
Sweet syndrome with differentiation syndrome related to Enasidenib
}

\author{
Amr Mohamed ${ }^{1}$ \\ ${ }^{1}$ Rochester Regional Health
}

January 5, 2021

\begin{abstract}
Rash is frequently seen in patients with leukemia either as a sequence of thrombocytopenia, or as result of infection or allergic drug rash, however sometimes rash is pointing towards fatal complications of underlying drug therapy or underlying disease , not being aware of these alarming signs can have fatal consequences.
\end{abstract}

Title : Sweet syndrome with differentiation syndrome related to Enasidenib

Author : Mohamed Amr, MD

Affiliation : Department of Internal Medicine, Rochester General Hospital, Rochester, NY.

Corresponding Author: Mohamed Amr, MD

Contact number : 718-764-7202

Affiliation address : 1425 Portland Avenue, Rochester, NY, 14621.

Email address : amrelwagdycardiol@gmail.com

Funding source : none

Conflict of interest : none

Article type : Clinical image

Key words : Differentiation syndrome, Enasidenib, Sweet syndrome.

Key clinical message: Multiple conditions as Drug rash, thromboembolism, sepsis and heart failure can mimic differentiation syndrome so keeping high index of suspicion is important as the prognosis if treated is usually good and fatal if missed.

Word count : 255 words

\section{Figures: 1}

65-year-old female who was admitted to hospital for treatment of acute myeloid leukemia, she was on Enasidenib for IDH2+ mutation in addition to standard leukemia therapy, after one week of therapy she started having fever up to $102 \mathrm{~F}$ with generalized skin rash, her physical exam was normal except for progressively worsening generalized skin rash as shown in figure $1 \mathrm{~A}$.

The initial dilemma in her diagnosis was whether this is infectious rash as she was neutropenic with absolute neutrophilic count (ANC) of 0 , she was started on cefepime followed by vancomycin, the next day her rash continued to worsen with new shortness of breath, her chest x-ray showed diffuse pulmonary infiltrates 
as shown in figure 1B and ANC did rapidly rise to 5 and now the clinical picture is more suggestive of differentiation syndrome with sweet syndrome.

She was a started on dexamethasone with resolution of the shortness of breath, fever, lung infiltrates and skin rash, skin biopsy was performed and it had shown prominent edema in the superficial dermis with dense infiltrate of neutrophils in the upper and mid-dermis with endothelial swelling and no evidence of vasculitis all consistent with sweet syndrome.

The main message is that differentiation syndrome happen in $20 \%$ of patients receiving isocitrate dehydrogenase inhibitor[1], it is sometimes associated with sweet syndrome, missing the diagnosis lead to fatal complications and the presentation can mimic drug rash, thromboembolism, infection or heart failure so keeping high index of suspicion is important as the prognosis if treated is usually good $[1,2]$.

\section{References}

1 - Fathi AT, DiNardo CD, Kline I, et al. Differentiation Syndrome Associated With Enasidenib, a Selective Inhibitor of Mutant Isocitrate Dehydrogenase 2: Analysis of a Phase 1/2 Study. JAMA Oncol. 2018 Aug 1; 4(8):1106-1110. doi: 10.1001/jamaoncol.2017.4695. PMID: 29346478; PMCID: PMC5885269.

2 - DiNardo CD, Stein EM, de Botton S, et al. Durable Remissions with Ivosidenib in IDH1-Mutated Relapsed or Refractory AML. N Engl J Med. 2018 Jun 21; 378(25):2386-2398. doi: 10.1056/NEJMoa1716984. Epub 2018 Jun 2. PMID: 29860938.

\section{Figure legends:}

Figure 1A: showing generalized papulovesicular skin rash on the anterior abdominal wall.

Figure 1B: showing chest x-ray with evidence of bilateral diffuse interstitial lung infiltrates, no cardiomegaly.

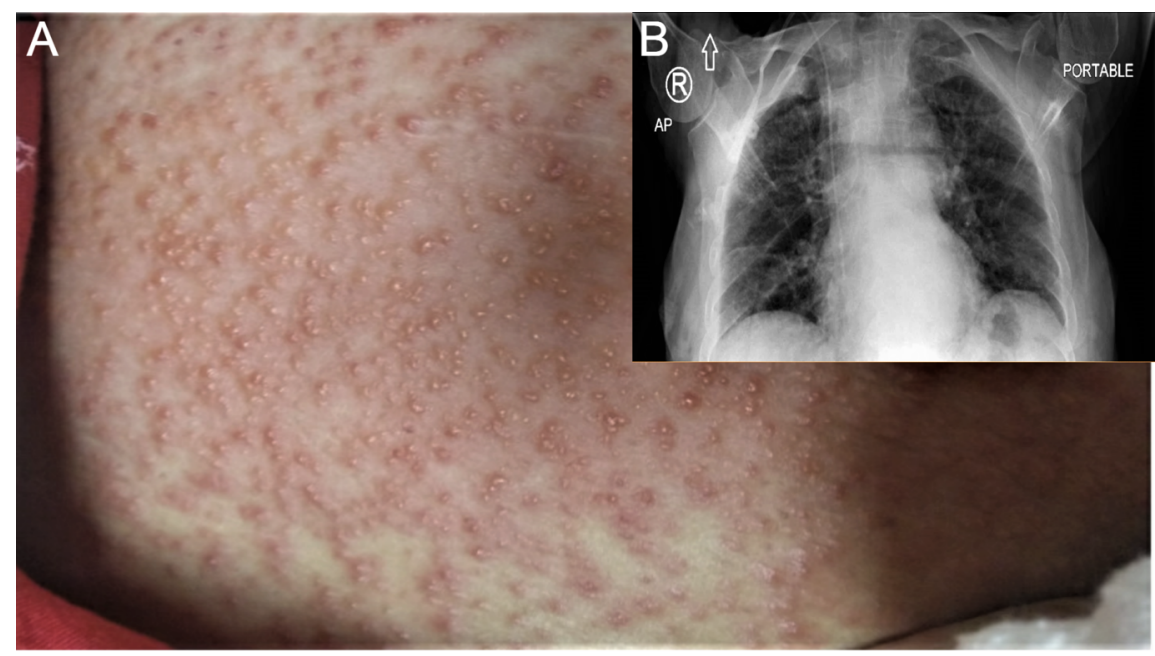

